This item was submitted to Loughborough's Research Repository by the author.

Items in Figshare are protected by copyright, with all rights reserved, unless otherwise indicated.

\title{
Superconducting analogs of quantum optical phenomena: macroscopic quantum superpositions and squeezing in a superconducting quantum- interference device ring
}

\section{PLEASE CITE THE PUBLISHED VERSION}

http://dx.doi.org/10.1103/PhysRevA.69.043804

\section{PUBLISHER}

(C) American Physical Society

\section{VERSION}

VoR (Version of Record)

\section{LICENCE}

CC BY-NC-ND 4.0

\section{REPOSITORY RECORD}

Everitt, Mark J., T.D. Clark, P.B. Stiffell, A. Vourdas, J.F. Ralph, R.J. Prance, and H. Prance. 2019. "Superconducting Analogs of Quantum Optical Phenomena: Macroscopic Quantum Superpositions and Squeezing in a Superconducting Quantum-interference Device Ring”. figshare. https://hdl.handle.net/2134/12069. 
This item was submitted to Loughborough's Institutional Repository (https://dspace.lboro.ac.uk/) by the author and is made available under the following Creative Commons Licence conditions.

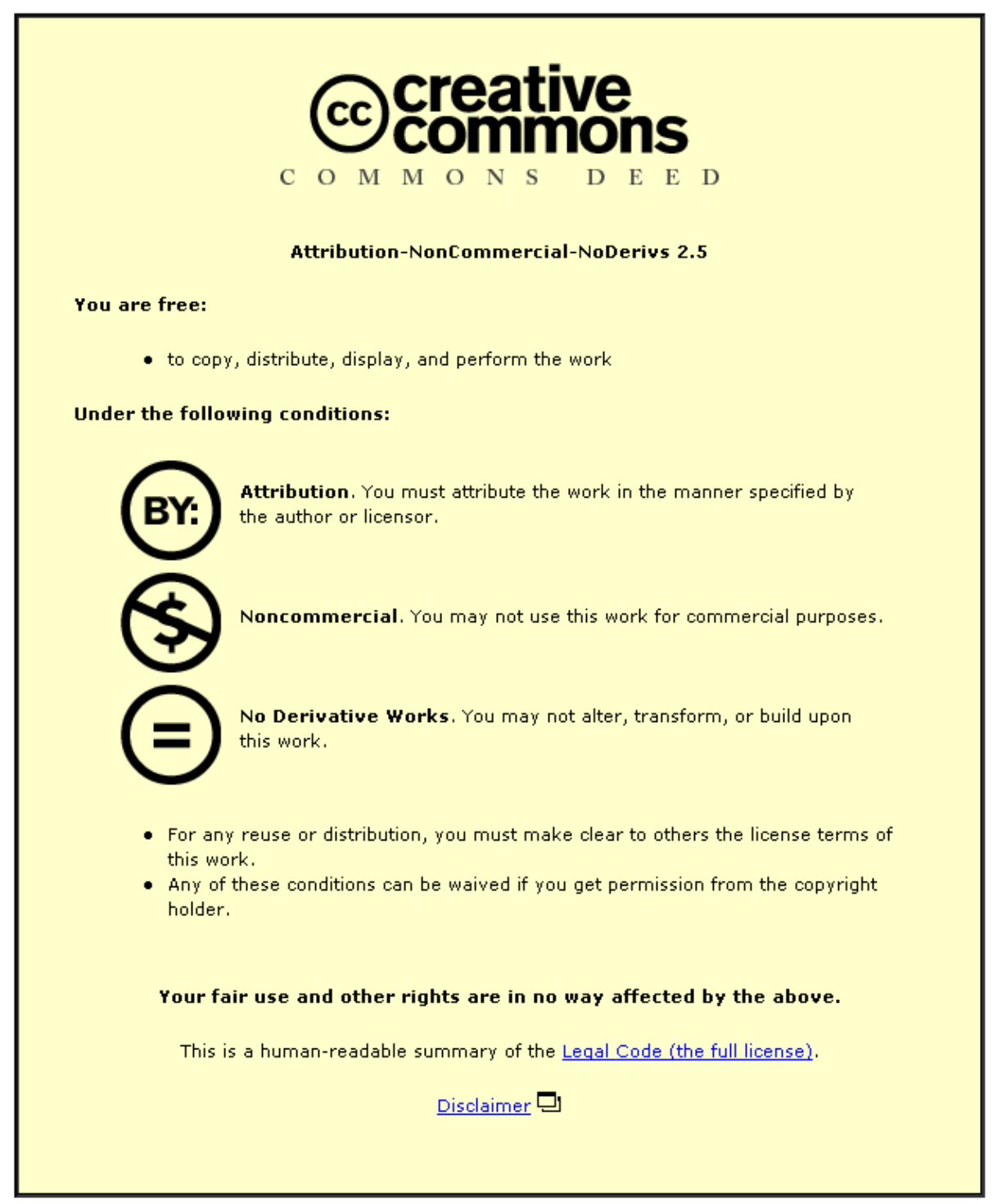

For the full text of this licence, please go to: http://creativecommons.org/licenses/by-nc-nd/2.5/ 


\title{
Superconducting analogs of quantum optical phenomena: Macroscopic quantum superpositions and squeezing in a superconducting quantum-interference device ring
}

\author{
M. J. Everitt, ${ }^{1, *}$ T. D. Clark, ${ }^{1, \dagger}$ P. B. Stiffell, ${ }^{1}$ A. Vourdas, ${ }^{2}$ J. F. Ralph, ${ }^{3}$ R. J. Prance,,${ }^{1}$ and H. Prance ${ }^{1}$ \\ ${ }^{1}$ Centre for Physical Electronics and Quantum Technology, School of Science and Technology, University of Sussex, Brighton, \\ Sussex BN1 9QT, United Kingdom \\ ${ }^{2}$ Department of Computing, Bradford University, Bradford, West Yorkshire BD7 1DP, United Kingdom \\ ${ }^{3}$ Department of Electrical and Electronic Engineering, Liverpool University, Brownlow Hill, Liverpool L69 3GJ, United Kingdom
}

(Received 23 August 2002; revised manuscript received 26 August 2003; published 5 April 2004)

\begin{abstract}
In this paper we explore the quantum behavior of a superconducting quantum-interference device (SQUID) ring which has a significant Josephson coupling energy. We show that the eigenfunctions of the Hamiltonian for the ring can be used to create macroscopic quantum superposition states of the ring. We also show that the ring potential may be utilized to squeeze coherent states. With the SQUID ring as a strong contender as a device for manipulating quantum information, such properties may be of great utility in the future. However, as with all candidate systems for quantum technologies, decoherence is a fundamental problem. In this paper we apply an open systems approach to model the effect of coupling a quantum-mechanical SQUID ring to a thermal bath. We use this model to demonstrate the manner in which decoherence affects the quantum states of the ring.
\end{abstract}

DOI: 10.1103/PhysRevA.69.043804

PACS number(s): 42.50.Ct, 42.50.Dv, 85.25.Dq, 74.50.+r

\section{INTRODUCTION}

In two recent publications [1,2] we reported on the theoretical description of a quantum-mechanical superconducting quantum-interference device (SQUID) ring (here, a thick superconducting ring enclosing a single Josephson weak link device) coupled to quantized electromagnetic field (em) oscillator modes. In this work we emphasized that the SQUID ring could be used to control various quantum phenomena involving each of the circuit components of the coupled system via the static magnetic bias flux $\Phi_{x}$ applied to the ring. These included frequency conversion between the em modes and quantum entanglement extending across the system, both with relevance to emerging quantum technologies based on Josephson devices [3-13]. Furthermore, work by Friedman et al. on SQUID rings has highlighted another phenomenon of potentially great significance to these incipient technologies, namely, the creation of externally controlled superpositions of macroscopically distinct states in a SQUID ring, or other, Josephson weak link based, circuit configurations [14]. As will become apparent, the creation and control of such states is a natural application for a SQUID ring.

In this paper we consider the creation and control of macroscopic quantum superposition states in a SQUID ring alone, uncoupled to any em oscillator modes. First, we consider the spectral properties of the ring Hamiltonian. Then we observe that at certain points in the bias flux $\Phi_{x}$ applied to the ring the eigenfunctions of this Hamiltonian form macroscopic superposition states. We show that a strong enough level of dissipation may destroy the quantum nature of these

\footnotetext{
*Electronic address: m.j.everitt@sussex.ac.uk

†Electronic address: t.d.clark@sussex.ac.uk
}

states, while leaving the flux in the SQUID ring in a statistical mixture of two macroscopically distinguishable states. Following this we demonstrate that a SQUID ring can be used to create (form) a controllable macroscopic superposition of states. In addition, we show that a SQUID ring with a sufficiently large Josephson coupling term in its potential can be used to squeeze coherent states. In this it is apparent that physical phenomena associated with SQUID rings, and with quantum circuits built around SQUID rings, have analogies with effects well known in the field of quantum optics. Indeed, the SQUID ring can be viewed as a nonlinear medium which, for example, can be utilized to generate entanglements, frequency conversion, superposition states, and squeezing. However, the SQUID ring has significant advantages over the generally weakly polynomial nonlinear media of quantum optics which are usually weakly coupled to external em fields. Thus, it is extremely nonperturbative in nature (and concomitantly capable of inducing extremely nonlinear behavior $[1,2,15]$ ) with a coupling to em modes that can be adjusted by means of an external bias flux. This would appear to make the SQUID a prime candidate for future developments in what is, in effect, highly nonperturbative quantum optics, albeit at much lower frequencies. In practice, these frequencies would typically be much less than $1 \mathrm{THz}$ for low critical temperature superconductors.

\section{BACKGROUND}

Wigner and Weyl functions

Although the Wigner and Weyl functions are familiar to those working in the field of quantum optics [16-18], their use in the quantum description of Josephson weak link circuits, and in particular SQUID rings, appears to be rather 




FIG. 1. Illustrative example of the (a) Wigner and (b) absolute value of the Weyl functions of the first four energy eigenstates of the simple harmonic oscillator (increasing in energy from left to right).

limited. The Wigner function is defined to be

$$
\begin{aligned}
W(x, p) & =\frac{1}{2 \pi} \int d \zeta\left\langle x+\frac{1}{2} \zeta|\rho| x-\frac{1}{2} \zeta\right\rangle \exp (-i \zeta p) \\
& =\frac{1}{2 \pi} \int d \zeta\left\langle p+\frac{1}{2} \zeta|\rho| p-\frac{1}{2} \zeta\right\rangle \exp (-i \zeta x),
\end{aligned}
$$

where $\rho$ is the density operator describing the state of the system with conjugate variables position $x$ and momentum $p$. Physically, the Wigner function can, to some extent, be considered as a generalization of the wave function of the quantum system under study in which we are provided with information in both position and momentum space. We note that the Wigner function may, and often does, take on negative as well as positive values. An important and characteristic feature of the Wigner function is that the quantum correlations between the macroscopically distinct components of a macroscopic superposition state can be seen in an obvious and graphical way, i.e., these correlations will appear in the Wigner function as interference terms between the states of the superposition in the $x-p$ phase plane.

By contrast, the Weyl function is defined as

$$
\begin{aligned}
\tilde{W}(X, P) & =\frac{1}{2 \pi} \int d \zeta\left\langle\zeta+\frac{1}{2} X|\rho| \zeta-\frac{1}{2} X\right\rangle \exp (-i \zeta P) \\
& =\frac{1}{2 \pi} \int d \zeta\left\langle\zeta+\frac{1}{2} P|\rho| \zeta-\frac{1}{2} P\right\rangle \exp (-i \zeta X) .
\end{aligned}
$$

It is apparent here that the Weyl function of a state is equal to the overlap of the displaced state with the original state so that $X$ and $P$ are considered as increments in position and momentum. As can be seen, the Weyl function is a generalized autocorrelation function; it is also the two-dimensional Fourier transform of the Wigner function. Thus, just as the Wigner function highlights the regions in the $x-p$ plane where the wave-function amplitude is significant, the Weyl function tells us where there exists a significant amplitude for correlations between intervals of $\Delta x(=X)$ and $\Delta p(=P)$ in this plane. A more detailed discussion and review of Wigner and Weyl functions, and the relationship between them, can be found in the literature $[18,19]$.

For those unfamiliar with these functions we provide a specific example in Fig. 1. Here, we have plotted the Wigner functions for the first four energy eigenstates of the simple harmonic oscillator together with the absolute values of their associated Weyl functions. We note that the Weyl function is, in general, complex valued. In this paper, therefore, we only ever plot its absolute value since, for our purposes, this provides us with sufficient information about the correlations of the wave function.

\section{The SQUID ring Hamiltonian}

Over the last two decades SQUID rings, viewed as single, macroscopic, quantum objects, have been the subject of considerable attention theoretically. In early studies the focus was primarily on time-independent properties and the interaction of SQUID rings with external environments $[15,20,21]$. Of late there has been much interest in timedependent behavior, for example, in solving the timedependent Schrödinger equation for a SQUID ring in the presence of a microwave field [22,23]. Recently, significant efforts have been devoted to the experimental measurement and control of macroscopic quantum superposition states in SQUID rings [14]. In this paper we proceed to develop a theoretical description of macroscopic quantum superpositions in SQUID rings, borrowing techniques that are commonly used in quantum optics. We extend the usefulness of this description by considering quantum-mechanical squeezed states in SQUID rings. For both superposition of states and squeezing in these rings we also discuss the effect of dissipation (decoherence).

In the widely used lumped component model of a SQUID ring $[15,20]$ the Hamiltonian takes the form

$$
H=\frac{Q^{2}}{2 C}+\frac{\left(\Phi-\Phi_{x}\right)^{2}}{2 \Lambda}-\hbar \nu \cos \left(\frac{2 \pi \Phi}{\Phi_{0}}\right)
$$

where $\Phi$ and $Q$ are, respectively, the magnetic flux threading the ring and the electric displacement flux across the weak link (with $[\Phi, Q]=i \hbar$ ), $\hbar \nu / 2$ is the matrix element for Josephson tunneling through the weak link (with critical current $\left.I_{c}=2 e \nu\right), \Phi_{0}=h / 2 e$ is the superconducting flux quantum, and $\Lambda$ and $C$ are, respectively, the ring inductance and the capacitance of the weak link in the ring.

Introducing a unitary translation operator $T$ $=\exp \left(-i \Phi_{x} Q / \hbar\right)$, we can then write down the ring Hamiltonian as

$$
H^{\prime}=\mathbb{T}^{\dagger} H \mathbb{T}=\frac{Q^{2}}{2 C}+\frac{\Phi^{2}}{2 \Lambda}-\hbar \nu \cos \left(2 \pi \frac{\Phi+\Phi_{x}}{\Phi_{0}}\right),
$$

where it is clear that as $\hbar \nu \rightarrow 0$ the system behavior reduces to that of a simple harmonic oscillator. Given the relation between our system and the simple harmonic oscillator, we now define creation and annihilation operators in the usual way, i.e., as

$$
a=\sqrt{\frac{C \omega}{2 \hbar}}\left(\Phi-\frac{i}{C \omega} Q\right), \quad a^{\dagger}=\sqrt{\frac{C \omega}{2 \hbar}}\left(\Phi+\frac{i}{C \omega} Q\right) .
$$

These raising and lowering operators, as used in quantum optics, then allow us to write the ring Hamiltonian in a more convenient form. We also choose to express it in dimension- 
less units, normalized to $\hbar \omega$, where $\omega / 2 \pi=1 / 2 \pi \sqrt{\Lambda C}$ is the SQUID ring oscillator frequency. This takes the form

$$
\mathcal{H}=\left(a^{\dagger} a+\frac{1}{2}\right)-\frac{\nu}{\omega} \cos \left(\frac{2 \pi}{\Phi_{0}} \sqrt{\frac{\hbar}{2 C \omega}}\left[a+a^{\dagger}\right]+2 \pi \varphi_{x}\right),
$$

where $\varphi_{x}=\Phi_{x} / \Phi_{0}$ is the normalized static bias flux applied to the SQUID ring. We note that the cosine term in the Hamiltonian introduces nonlinearities to all orders. We have seen that this property of the SQUID ring introduces highly nonperturbative effects $[1,2]$ when coupled to other circuit systems. In this paper we show that it also gives rise to quantum superpositions of macroscopically distinct states and squeezing within the ring itself.

From Eq. (3) it is apparent that as long as the ratio $\nu: \omega$ and the product $C \omega(=\sqrt{C / \Lambda})$ remain the same the physics of this system is unchanged. We therefore choose values of $C$ and $\hbar \nu$ (or equivalently $I_{c}=2 e \nu$ ) that can be attained using currently available microfabrication techniques that are physically sensible and that will lead to SQUID ring systems exhibiting quantum behavior at experimentally accessible temperatures. With these factors in mind we choose the circuit parameters $C=5 \times 10^{-15} \mathrm{~F}, \Lambda=3 \times 10^{-10} \mathrm{H}$, and a sufficiently large value of $\hbar \nu\left(=0.047 \Phi_{0}^{2} / \Lambda ; I_{c}=2 \mu \mathrm{A}\right)$ to generate clear wells in the ring potential. Thus, for a thin-film Josephson tunnel junction weak link with a $1 \mathrm{~nm}$ oxide insulator thickness (dielectric constant $\approx 10$ ) a capacitance of 5 $\times 10^{-15} \mathrm{~F}$ yields junction dimensions $\approx 0.25 \mu \mathrm{m}$ square, readily achieved using microfabrication. Again, with these dimensions the supercurrent density in the junction is around $4 \mathrm{kA} \mathrm{cm}^{-2}$ which is perfectly reasonable. Furthermore, with $C=5 \times 10^{-15} \mathrm{~F}$ and $\Lambda=3 \times 10^{-10} \mathrm{H}, \omega / 2 \pi=130 \mathrm{GHz}$, well below the frequency corresponding to the superconducting energy gap in niobium $(\approx 1 \mathrm{THz})$, a metal often used in weak link device fabrication. Given these chosen parameter values, and assuming, as our example, SQUID circuits based on niobium, these correspond to $\nu / \omega=7.9$ and $C \omega=4.1$ $\times 10^{-3}$, values which, unless otherwise stated, we now keep to throughout this paper.

Adopting these values of $\Lambda, C$, and $\hbar \nu$ we show in Fig. 2 the SQUID ring potential $U\left(\Phi, \Phi_{x}\right)=\left(\Phi-\Phi_{x}\right)^{2} / 2 \Lambda$ $-\hbar \nu \cos \left(2 \pi \Phi / \Phi_{0}\right)$ - see Eq. (1) - computed for three different values of $\Phi_{x}\left[=0 \Phi_{0}\right.$ (top), $0.49 \Phi_{0}$ (middle), and $0.5 \Phi_{0}$ (bottom)]. We also show in this figure the probability densities of the wave functions of the ring as a function of $\Phi$. These probability densities are displaced by their energy eigenvalues, found by solving the time-independent Schrödinger equation using the Hamiltonian (1). As the bottom of each of the local wells in the ring potential in Fig. 2 is approximately quadratic, we would expect the solutions deep within these wells to look like those for the simple harmonic oscillator. In addition, we find that, on average, these states are slightly squeezed in terms of the magnetic flux variable $\Phi$. For example, in Fig. 2 the lowest state in each of the wells for $\Phi_{x}=0.49 \Phi_{0}$ has $[\Delta(\sqrt{C \omega / \hbar} \Phi)]^{2}$ $\approx 0.43$ and $[\Delta(\sqrt{1 / C \hbar \omega} Q)]^{2} \approx 0.58$ (compared to 0.5 in the unsqueezed state), where $\Phi$ and $Q$ are normalized to
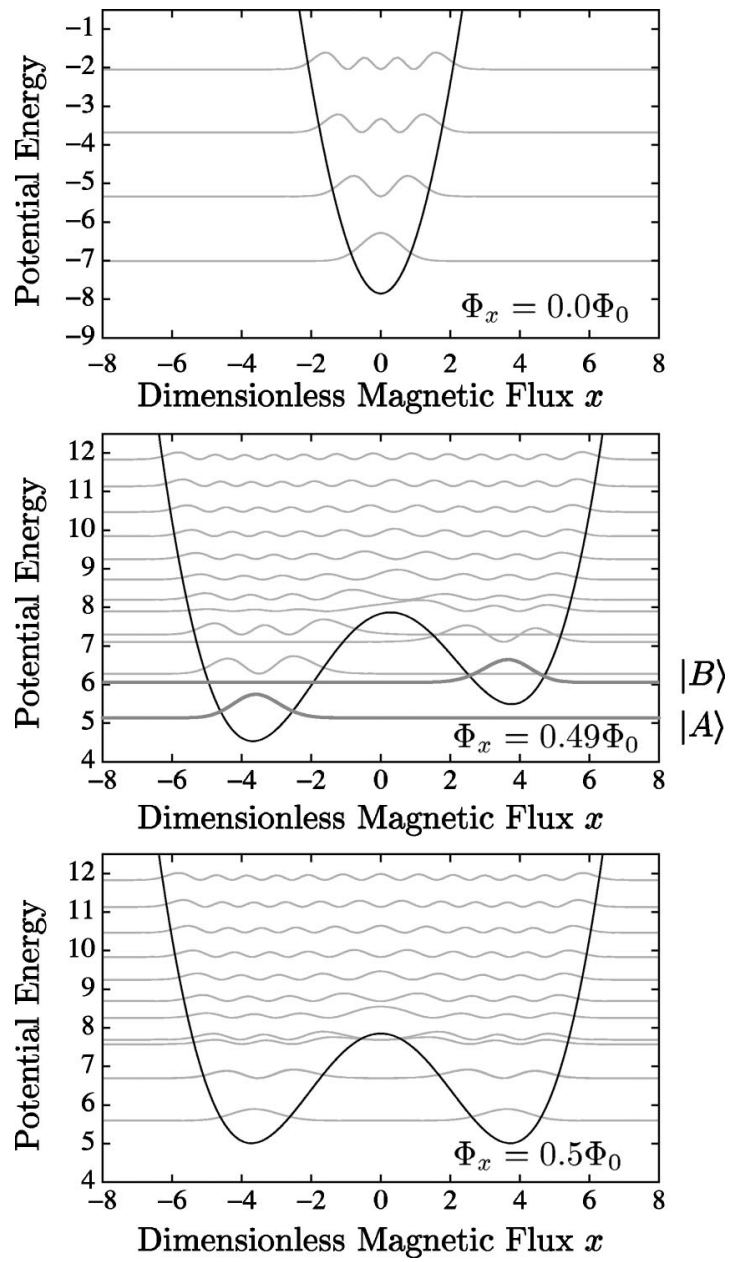

FIG. 2. Potential energy in units of $\hbar \omega(\omega=1 / \sqrt{\Lambda C})$ vs dimensionless flux [see Eq. (4)] for a SQUID ring with parameter values $C=5 \times 10^{-15} \mathrm{~F}, \quad \Lambda=3 \times 10^{-10} \mathrm{H}$, and $\hbar \nu=0.047 \Phi_{0}^{2} / \Lambda$ for $\Phi_{x}$ $=0 \Phi_{0}, 0.49 \Phi_{0}$, and $0.5 \Phi_{0}$. Also shown are the probability density functions of the ring wave functions displaced by their energy eigenvalues.

$(\hbar / C \omega)^{1 / 2}$ and $(\hbar C \omega)^{1 / 2}$, respectively. To aid in the description of the system we adopt the term locally $s$ harmonic to describe this behavior, where the prefix $s$ denotes the squeezed nature of these states. The equivalence of the lowlying set of energy eigenvalues deep in each (local) well is a sound approximation except where the energy levels of two or more wells align. In such cases, of course, symmetric and antisymmetric superpositions of the eigenfunctions for the isolated wells develop.

In Fig. 3 we show a set of computed SQUID ring energy eigenvalues of the Hamiltonian operator (3), starting with the ground state, as a function of external flux $\Phi_{x}$ and spanning one flux quantum. For clarity the energy levels are shown as alternating black and gray lines. As can be seen, for the values of $\Lambda, C$, and $\hbar \nu$ we have adopted in this work, there exist many crossing points of the original eigenvalues as $\Phi_{x}$ is changed over a $\Phi_{0}$ period. At the scale provided in Fig. 3 these crossing points appear to be degenerate in energy (e.g., in the lowest two eigenvalues at $\Phi_{x}=0.5 \Phi_{0}$ ). Of course, this is not the case, as would be evident if these crossing points 


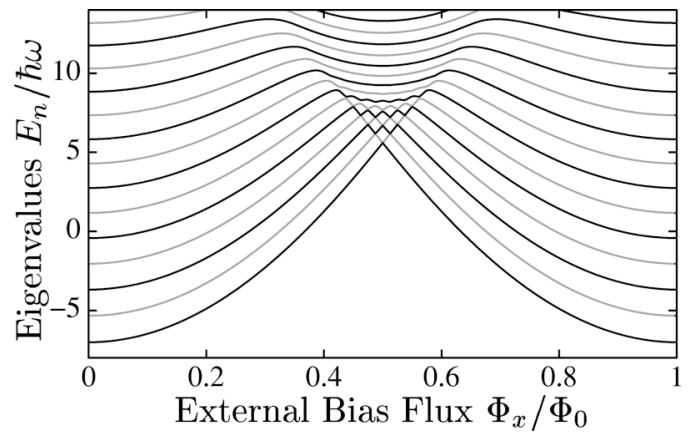

FIG. 3. Computed SQUID ring energy eigenvalues of the Hamiltonian for the SQUID ring of Fig. 2.

were computed to sufficient accuracy. However, for the potential wells shown in Fig. 2, with the very weak coupling between levels in different wells, the energy splittings at these points may be extremely small indeed.

\section{RESULTS}

\section{Quantum superpositions of macroscopically distinct states}

Ignoring, for the moment, the special cases where the locally $s$-harmonic oscillator states are degenerate (zero coupling between wells) we instead consider making an equal superposition of two energy eigenstates. We start by assuming that these two states are locally $s$ harmonic in different wells and, for simplicity, take these states to correspond to the lowest-energy levels in each well. Then, as one would expect, we create a superposition of macroscopically distinct states in flux. As this superposition state is no longer an eigenstate of the Hamiltonian for the system, it must evolve with time. This evolution introduces a phase difference between the two stationary (locally $s$-harmonic) states in the superposition which manifests itself in a time-dependent evolution of the interference term in the Wigner function.

In computing Wigner functions for the SQUID ring it is convenient to introduce equivalent dimensionless position $x$ and momentum $p$ operators in place of $\Phi$ and $Q$. These are defined as

$$
x=\sqrt{\frac{C \omega}{\hbar}} \Phi, \quad p=\sqrt{\frac{1}{\hbar C \omega}} Q .
$$

An example of a macroscopic superposition state in a SQUID ring, as illustrated through its computed Wigner function, is shown in Fig. 4, both in perspective and in projection on the $x$ - $p$ plane. Here, we have taken our standard values of $\Lambda, C$, and $\hbar \nu$ (above) and have selected the state $(1 / \sqrt{2})(|A\rangle+|B\rangle)$ at the flux bias $\Phi_{x}=0.49 \Phi_{0}$ of Fig. 2(b). In this example we can distinguish in the Wigner function two macroscopically distinct flux states of the SQUID ring in the $x-p$ plane (i.e., the Gaussian-like components), separated from one another by an oscillatory region. The latter, oscillatory, region arises because of the quantum coherence between the two separate components in the superposition and demonstrates that we are indeed dealing with a true quantum superposition of macroscopically distinct states in flux. We
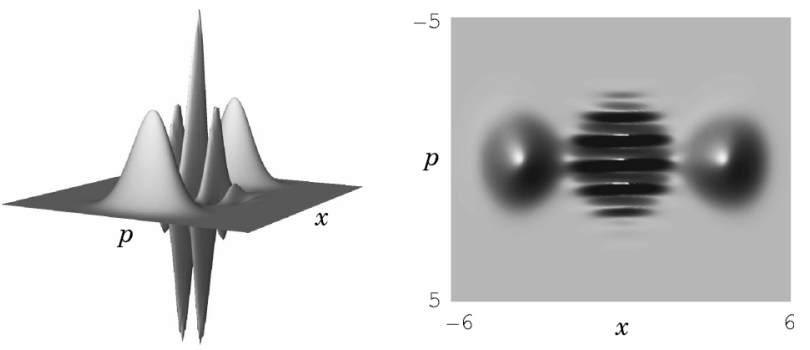

FIG. 4. Wigner function showing, both in perspective and in projection onto the $x-p$ plane, the nature of this quantum superposition of macroscopically distinct states $(1 / \sqrt{2})(|A\rangle+|B\rangle)$ for the SQUID ring of Fig. 2. The dimensionless quantities $x$ and $p$ are defined in Eq. (4).

note that the Wigner function of Fig. 4 has been calculated at a fixed time $t=0$. However, its general form does not vary with time. Nevertheless, there is dynamical evolution of the interference term in the superposition but not in the observable flux states.

\section{Tunable superposition of states in a SQUID ring}

We now consider the potential with a static bias flux $\Phi_{x}$ $=0.5 \Phi_{0}$, as shown in the bottom plot of Fig. 2 . By examining the first two energy eigenstates we find that we have wave functions which are a symmetric, $|s\rangle$, and an antisymmetric, $|a\rangle$, superposition of the lowest energy, locally $s$-harmonic, oscillator vacuum states of the two middle wells. These two states, which are squeezed, are extremely close in energy due to the height of the barrier between the wells in the potential. Even so, the interference terms in the Wigner function between the states for each of these macroscopically distinct states are $\pi / 2$ out of phase. However, from the viewpoint of theory we might also consider an equal superposition of these states of the form

$$
\frac{1}{\sqrt{2}}[|s\rangle+\exp (i \theta)|a\rangle]
$$

where $\theta$ is the phase. Let $E_{s}$ and $E_{a}$ be the eigenenergies corresponding to the eigenstates $|s\rangle$ and $|a\rangle$ so that $\Delta E=E_{s}$ $-E_{a}$ the energy splitting associated with the lifting of the degeneracy concomitant with tunneling between the adjacent wells. Then $\theta=\Delta E t$. It is seen that this phase $\theta$ changes as a function of time and any particular value of $\exp (i \theta)$ is realized cyclically with period $2 \pi / \Delta E$. If we wished to fix our state in a given superposition, we can do this simply by changing our external magnetic bias flux by a fraction. If this fractional change in bias flux is sufficient, this will remove the coupling between the wells and set $\theta$. Alternatively, this could be achieved by tuning the barrier height of the SQUID ring (see Fig. 9 later in this paper) so that the Josephson-tunneling energy becomes vanishingly small [24]. The effect of changing $\theta$ can be dramatic, as illustrated in Fig. 5, where the Wigner function in the $x-p$ plane is shown for selected values of this parameter. As is apparent, when the wave function for the ring is strongly (but not completely) localized in two or more 


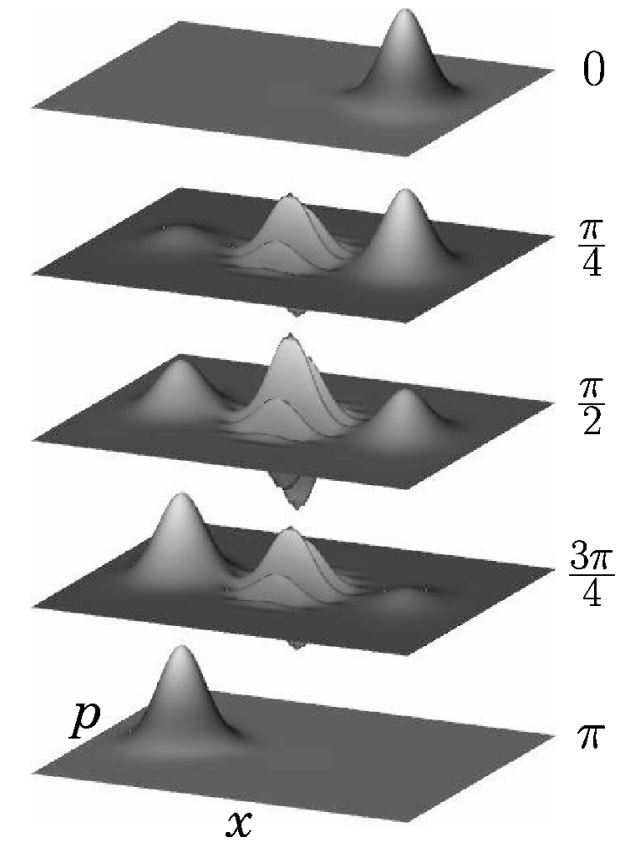

FIG. 5. Wigner function of a superposition of the lowest two (symmetric and antisymmetric) energy eigenstates as a function of phase-see Eq. (5) and related text and Fig. 4 for scalings.

regions in the ring potential, it is the quantum interaction between these regions that is responsible for the creation of superposition of macroscopically distinct states.

We note that given the very slightly different energies between the symmetric and antisymmetric superposition states, due to the height of the barrier between the two wells, the superposition (5) will oscillate slowly back and forth between these wells. Using these energies we calculate this period to be $100 \mathrm{~ns}$. This is much longer than the time constant corresponding to the $\Lambda C$ frequency of the SQUID ring in our example (i.e., for $C=5 \times 10^{-15} \mathrm{~F}, \Lambda=3 \times 10^{-10} \mathrm{H}$ this is $7.6 \mathrm{ps}$ ) but well within the decoherence times of modern SQUID ring circuits [4,25-27].

In the above discussion we have considered the development of macroscopic superposition states in a SQUID ring based on a choice of ring parameters which can be realized by fabrication and which are physically reasonable. However, this choice does not connect directly with published experimental data. We will therefore deviate briefly from our standard parameter values and consider an explicit example of superpositions of SQUID ring states as reported recently in the literature by Friedman et al. [14]. In this paper the authors considered a quantum superposition of distinct macroscopic states and presented experimental evidence, indicating that a SQUID ring could be placed into a superposition of two magnetic flux states. We now demonstrate by computation that this superposition may indeed form a true macroscopic superposition state. The experimental system used a SQUID ring with the circuit parameters $C=1.03 \times 10^{-13} \mathrm{~F}$, $\Lambda=2.38 \times 10^{-10} \mathrm{H}$, and $I_{c}=2.02 \times 10^{-6} \mathrm{~A}$. To obtain a superposition state as demonstrated in this paper we used the external bias flux quoted by the authors, i.e., $\Phi_{x}$ $=0.514466 \Phi_{0}$. The potential energy of a SQUID ring with these parameters is shown in Fig. 6 together with the prob-

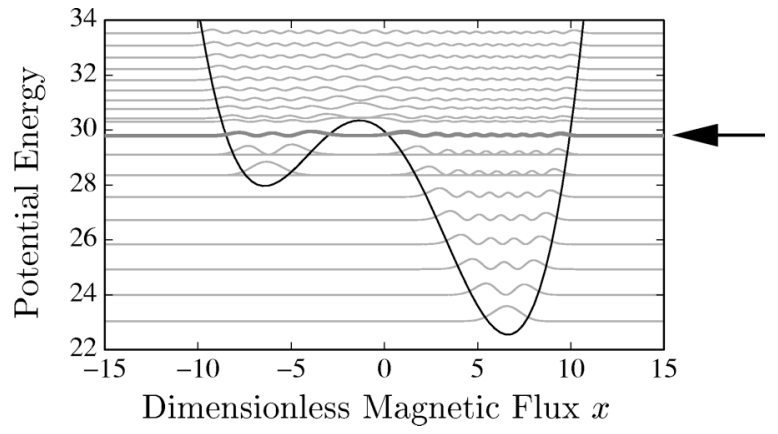

FIG. 6. Potential energy in units of $\hbar \omega$ vs dimensionless flux [see Eq. (4)] of a SQUID ring with parameter values $C=1.03$ $\times 10^{-13} \mathrm{~F}, \quad \Lambda=2.38 \times 10^{-10} \mathrm{H}, \quad I_{c}=2.02 \times 10^{-6} \mathrm{~A}, \quad$ and $\Phi_{x}$ $=0.514466 \Phi_{0}$ (after Friedmann et al. [14]). Also shown are the probability density functions of the ring's wave functions displaced by their energy eigenvalues. The arrow indicates the states used to calculate the superposition state.

ability densities for the ring wave functions. These are displaced, as before, by their energy eigenvalues. In this figure we have marked with an arrow the states in the left-hand and right-hand wells from which we will form our superpositions. These are the states that were utilized in the experiment of Friedman et al.

These eigenfunctions are similar to those of Fig. 5, in that they form a symmetric, $|s\rangle$, and an antisymmetric, $|a\rangle$, superposition of the locally $s$-harmonic states of the separate wells, albeit with higher and different ordinal numbers. The quantum state of the SQUID ring, as reported by Friedman et al. [14], will thus be a superposition of these two eigenstates. For our purposes it is therefore sufficient to look (again) at superpositions of the form

$$
\frac{1}{\sqrt{2}}[|s\rangle+\exp (i \theta)|a\rangle] .
$$

In Fig. 7 we show the Wigner functions for three different superpositions of these eigenstates, again both in perspective and projection onto the $x$ - $p$ plane. We notice that compared with the Wigner functions of Fig. 5 both the states of the macroscopic quantum superposition corresponding to the left- and right-hand wells in Fig. 7 and the region of interference between them display more complex patterns. Given the choice of more highly excited states in the experiment of Friedmann et al., this is to be expected. Nevertheless, Fig. 7 demonstrates that the Wigner function (and, of course, the associated Weyl function) can expose sophisticated quantum coherent behavior in SQUID rings.

\section{Dissipation}

In considering the effect of dissipation on the calculations presented in this paper we have chosen to use a standard approach, and one familiar in quantum optics. This is to be done by coupling the SQUID ring to a decohering monochromatic thermal bath. The master equation for the evolution of the density operator of the system then takes the form [28] 

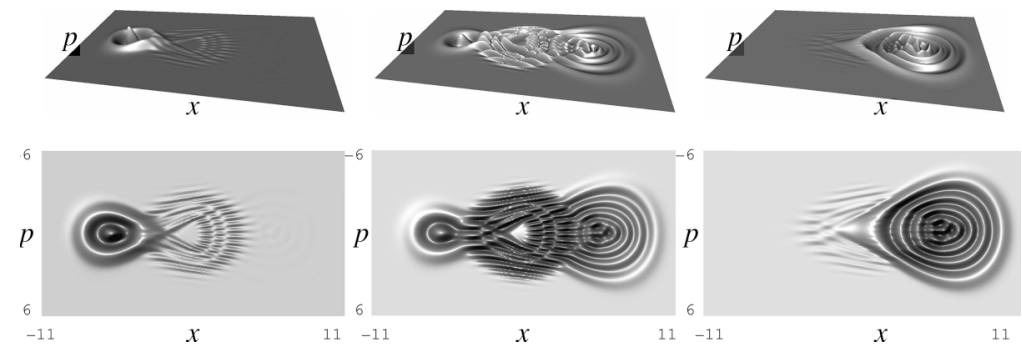

FIG. 7. Wigner function, from left to right, for the three distinct phase $0, \pi / 2$, and $\pi$ (cf. Fig. 5 and after Friedmann et al. [14], showing, both in perspective and in projection onto the $x$ - $p$ plane, the superposition of the (symmetric and antisymmetric) energy eigenstates as indicated in Fig. 6.

$$
\begin{aligned}
\frac{\partial \rho}{\partial t}= & -\frac{i}{\hbar}[\mathcal{H}, \rho]+\frac{\gamma}{2 \hbar}(M+1)\left(2 a \rho a^{\dagger}-a^{\dagger} a \rho-\rho a^{\dagger} a\right) \\
& +\frac{\gamma}{2 \hbar} M\left(2 a^{\dagger} \rho a-a a^{\dagger} \rho-\rho a a^{\dagger}\right),
\end{aligned}
$$

where $M$ is related to the temperature $T$ and the frequency $\omega_{b}$ of each decohering bath via $M_{i}=\left[\exp \left(\hbar \omega_{b} / k_{B} T\right)-1\right]^{-1}$ and $\gamma_{i}$ is the coupling (damping rate) between each of the components to its respective thermal bath. For the following examples that we will now calculate we set $T=1 \mathrm{~K}$ with $\omega_{b}=\omega$.

To illustrate the effect that dissipation has upon a macroscopic superposition state in a SQUID ring, we now solve the master equation (7) for the system evolution, where the ring is taken to be in its ground state at $\Phi_{x}=0.5 \Phi_{0}$. For this computation we return to our initial circuit values of $C=5$ $\times 10^{-15} \mathrm{~F}, \Lambda=3 \times 10^{-10} \mathrm{H}$, and $\hbar \nu\left(=0.047 \Phi_{0}^{2} / \Lambda\right)$. Here we have chosen to use a decoherence rate of $0.01 \omega$. In Fig. 8 we have plotted the Wigner and Weyl functions for the ring at particular times. As the system evolves we notice that the Wigner and Weyl functions display very clearly the disappearance of the quantum coherence between the two states of this superposition. We also note that after sufficient time has elapsed the Wigner function still displays two distinct flux probabilities. However, the SQUID ring is longer in a pure state and this represents a classical coin toss probability and not a quantum one. This is also clearly reflected in the disappearance of the symmetrically positioned correlation peaks in the Weyl function at these later times.

\section{Squeezed states of a SQUID ring}

Just as in quantum optics, where coherent light can be squeezed in the number-phase plane through its interaction with a nonlinear optical medium, we shall now demonstrate that a SQUID ring, with the cosine Hamiltonian (1), can be used to squeeze an initial coherent state. This starting condition in a SQUID ring can be achieved by changing the weak link structure in the ring. As is well known, the combined Josephson critical current in a parallel, two weak link, loop, connected by superconducting wires (a dc SQUID [29]), can be varied by adjusting the magnetic flux threading the loop. Such a structure can serve as the adjustable weak link within a larger diameter SQUID ring. This has been already discussed in several earlier papers, including that of Friedman et

al. [14]. The arrangement is depicted in Fig. 9.

Provided the control magnetic flux $\Phi_{x_{2}}$ threading the minor loop in Fig. 9 is large enough, and the weak links in the loop can be fabricated (in principle) to possess identical criti-

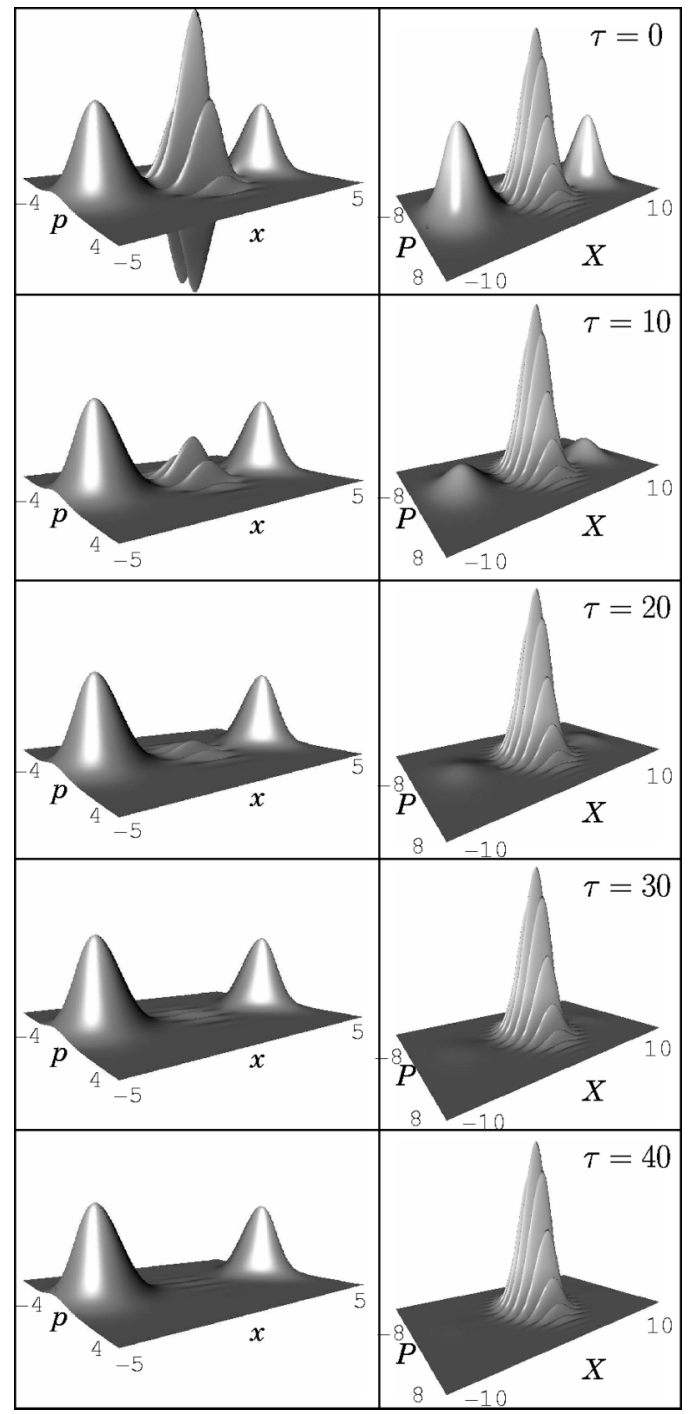

FIG. 8. Wigner (left) and Weyl (right) functions for the ground state of the SQUID ring of 3 evolving in the presence of dissipation ( $T=1 \mathrm{~K}$ and $\gamma=0.01 \omega$ ). The effect of dissipation is to remove the quantum correlations in this superposition of macroscopically distinct states over the time shown. 


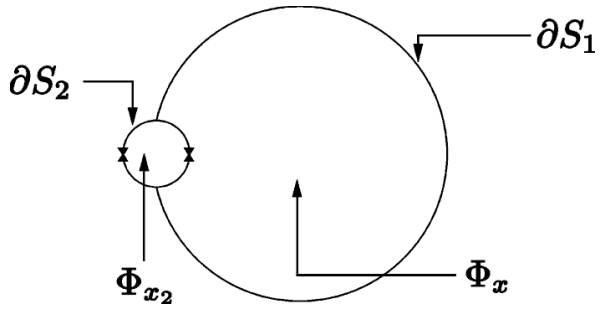

FIG. 9. Schematic of a SQUID ring with a (flux) controllable Josephson tunneling energy. Here the area enclosed by the outer loop $\partial S_{1}$ is much greater than that enclosed by the inner loop $\partial S_{2}$.

cal currents, the net supercurrent carried through the loop can be made vanishingly small. In this situation the Josephson coupling energy in Eq. (1) reduces essentially to zero leaving just the parabolic background term (i.e., as for a simple harmonic oscillator). Let us now consider a possible mechanism by which we could create a coherent state in a SQUID ring. If we allow the SQUID ring to decohere to its ground state, then, as we are in the low Josephson coupling limit, this state will very much resemble that of the vacuum state of the simple harmonic oscillator. Now, a coherent state is just the vacuum state displaced from the origin and then allowed to evolve freely. We can realize this for a SQUID ring by very rapidly changing the external bias flux so that the wave function does not change significantly in shape, but the relative expectation value of flux (position) no longer corresponds to the minimum of the potential well. We can, within reason, choose the ramp rate, start and end bias flux, to create a state which is a very good approximation to a coherent state at a required energy. We take this as the initial prepared condition of the major SQUID ring (with a main control flux $\Phi_{x_{1}}$ ) for which we can choose a coherent state $|A=i \sqrt{1}\rangle$. We follow this initial setup by a very rapid reduction in $\Phi_{x_{2}}$ to yield the desired (and finite) Josephson coupling energy in the principle SQUID ring corresponding to the ring potential considered throughout this paper. Clearly, in an experimental situation this reduction must take place on a time scale short compared with the decoherence time of the SQUID ring coupled to its environment. From the literature [4,25-27] it appears that the coherence times in SQUID rings can be sufficiently long to make this readily achievable. With this proviso the system is prepared in the required coherent state and then allowed to evolve over time using the master equation (7).

Here, as before, we take the SQUID ring circuit parameters to be $C=5 \times 10^{-15} \mathrm{~F}$ and $\Lambda=3 \times 10^{-10} \mathrm{H}$. However, in order to improve the effects of squeezing by the ring we have increased $\hbar \nu$ to $0.24 \Phi_{0}^{2} / \Lambda$, yielding a superconducting critical current density of around $20 \mathrm{kA} \mathrm{cm}^{-2}$. From our given initial condition, and by computing the uncertainties in flux, we see that the SQUID ring can squeeze the magnetic flux within the ring. We choose not to show the results for the charge as they do not provide any additional information relevant to this discussion. In Fig. 10 we show the uncertainty in flux for a selection of decoherence rates. Here, the horizontal line denotes that squeezing happens for $(\Delta x)^{2}$ less than $1 / 2$. We can clearly see that, on average, the ring has effectively squeezed this state. We also see that for a judi-

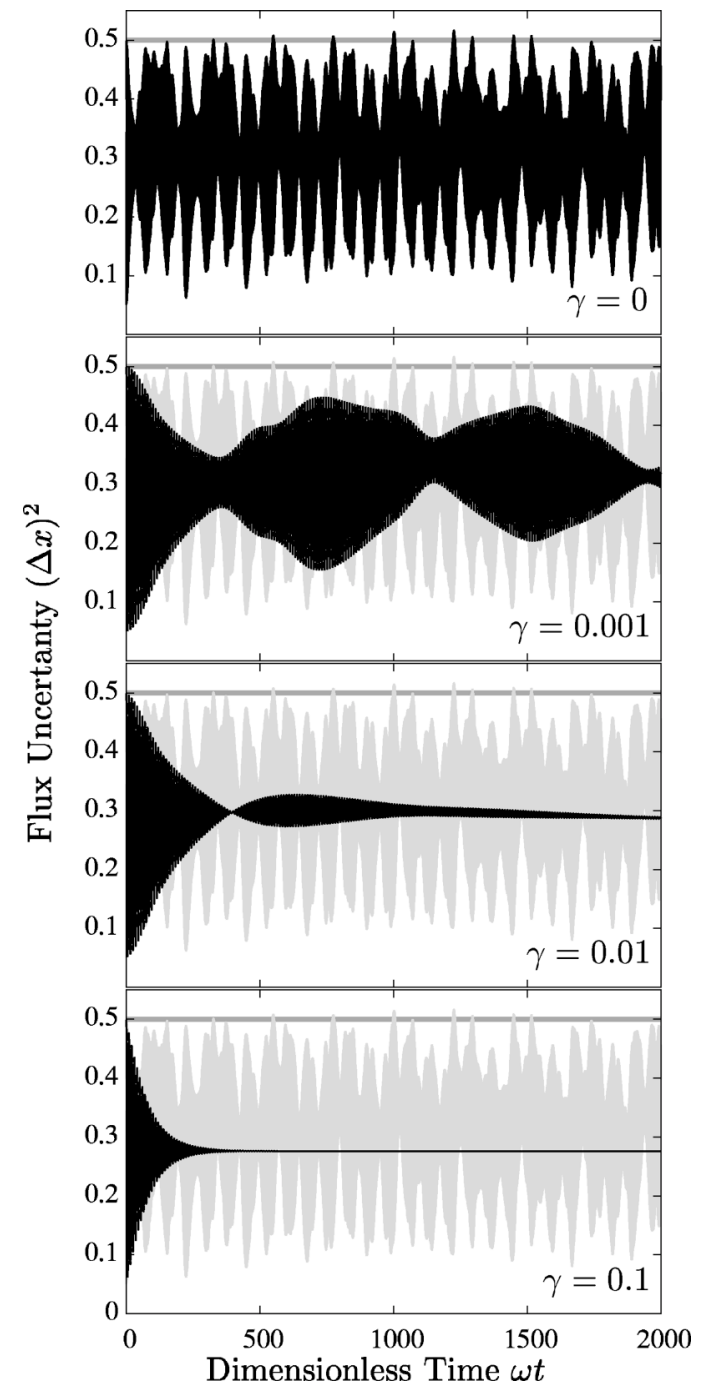

FIG. 10. Uncertainty in dimensionless flux [see Eq. (4)] vs dimensionless time $(\omega t)$, for a SQUID ring with parameter values $C$ $=5 \times 10^{-15} \mathrm{~F}, \Lambda=3 \times 10^{-10} \mathrm{H}$, and $\hbar \nu=0.24 \Phi_{0}^{2} / \Lambda$, computed for a selection of decoherence rates at $T=1 \mathrm{~K}$. Here the horizontal line denotes that squeezing occurs for $(\Delta x)^{2}$ less than $1 / 2$. The gray background oscillation for the $\gamma=0.001,0.01$, and 0.1 examples is the $\gamma=0$ pattern repeated for comparison.

cious choice of decoherence rate and interval of time, dissipation can assist in the squeezing process. We note that in the $\gamma=0.1$ case it can be seen that the SQUID ring decoheres to its (squeezed) vacuum state over a relatively short period. Thus, it appears that these potential wells may be used with facility to generate squeezing of the magnetic flux variable of the ring. It is therefore reasonable to assume that adiabatic changes of this potential would, unless matching conditions exist between local $s$-harmonic energy levels of adjacent wells, allow some adjustment of the expectation value of the flux in the ring.

\section{CONCLUSIONS}

In this paper we have explored two applications of SQUID rings, with strong analogies to the field of quantum 
optics, which may prove to be of great utility given the current interest in quantum circuit technologies. In the first we describe the manner in which we can create macroscopic superposition states in SQUID rings and the way these may be manipulated through an external control flux. In the second we provide a dynamical mechanism for inducing squeezing of the magnetic flux variable in a SQUID ring starting from an almost harmonic-oscillator state. These take advantage of the highly nonperturbative quantum nature of SQUID rings arising from the cosine coupling energy term in the ring Hamiltonian. As we emphasize, both are of great interest in the light of current research in the fields of quantum computing and quantum-information processing [10-13]. Moreover, the results presented in this paper emphasize that the SQUID ring can act as a versatile quantum device and, as we have shown previously, can be used to create correlations across extended, multicomponent, quantum circuit systems $[1,2,30-33]$. These correlations can, for example, be made manifest as quantum entanglements and quantum frequency up/down-conversion [1,2], with each controlled by the external bias flux applied to the SQUID ring. In this sense these phenomena, including macroscopic superposition states and squeezing, highlight the role of the SQUID ring as the essential machinery for a range of applications in superconducting quantum circuit technologies. This further enhances our understanding of these nonperturbative quantum objects and their possible usefulness in these incipient technologies.

\section{ACKNOWLEDGMENTS}

We would like to thank the EPSRC for its generous funding of this work and for sponsoring the U.K. Quantum Circuits Network. We would also like to express our thanks both to Professor C.H. van de Wal and Professor A. Sobolev for interesting discussions and to the Sussex High Performance Computing Initiative for the use of their NAG-IRIS explorer graphics software.
[1] M. J. Everitt, P. Stiffell, T. D. Clark, A. Vourdas, J. F. Ralph, H. Prance, and R. J. Prance, Phys. Rev. B 63, 144530 (2001).

[2] M. J. Everitt, T. D. Clark, P. Stiffell, H. Prance, R. J. Prance, A. Vourdas, and J. F. Ralph, Phys. Rev. B 64, 184517 (2001).

[3] I. Chiorescu, Y. Nakamura, C. Harmans, and J. Mooij, Science 299, 1869 (2003).

[4] J. Martinis, S. Nam, and J. Aumentado, Phys. Rev. Lett. 89, 117901 (2002).

[5] R. Rouse, S. Y. Han, and J. E. Lukens, Phys. Rev. Lett. 75, 1614 (1995).

[6] P. Silvestrini, B. B. Ruggiero, C. Granata, and E. Esposito, Phys. Lett. A 267, 45 (2000).

[7] Y. Nakamura, C. D. Chen, and J. S. Tsai, Phys. Rev. Lett. 79, 2328 (1997).

[8] Y. Nakamura, Y. A. Pashkin, and J. S. Tsai, Nature (London) 398, 786 (1999).

[9] C. H. van der Wal, A. C. J. ter Haar, F. K. Wilhelm, R. N. Schouten, C. J. P. M. Harmans, T. P. Orlando, S. Lloyd, and J. E. Mooij, Science 290, 773 (2000).

[10] H. Lo, S. Popescu, and T. Spiller, Introduction to Quantum Computation and Information (World Scientific, Teaneck, NJ, 1998).

[11] T. P. Orlando, J. E. Mooij, L. Tian, C. H. van der Wal, L. S. Levitov, S. Lloyd, and J. J. Mazo, Phys. Rev. B 60, 15398 (1999).

[12] Y. Makhlin, G. Schon, and A. Shnirman, Nature (London) 398, 305 (1999).

[13] D. V. Averin, Y. V. Nazarov, and A. A. Odintsov, Physica B 165, 945 (1990).

[14] J. R. Friedman, V. Patel, W. Chen, S. K. Tolpygo, and J. E. Lukens, Nature (London) 406, 43 (2000).

[15] T. Spiller, T. Clark, R. Prance, and A. Widom, Progress in Low
Temperature Physics (Elsevier Science, Amsterdam, 1992), Vol. XIII.

[16] E. Wigner, Phys. Rev. 40, 749 (1932).

[17] N. L. Balazs and B. K. Jennings, Phys. Rep. 104, 347 (1984).

[18] C. Chountasis and A. Vourdas, Phys. Rev. A 58, 848 (1998).

[19] W. Schleich, Quanum Optics in Phase Space (Wiley-VCH, Berlin, 2001).

[20] Y. Srivastava and A. Widom, Phys. Rep. 148, 1 (1987).

[21] A. J. Leggett, S. Chakravarty, A. T. Dorsey, M. P. A. Fisher, A. Garg, and W. Zwerger, Rev. Mod. Phys. 59, 1 (1987).

[22] T. D. Clark, J. Diggins, J. F. Ralph, M. Everitt, R. J. Prance, H. Prance, R. Whiteman, A. Widom, and Y. N. Srivastava, Ann. Phys. (N.Y.) 268, 1 (1998).

[23] J. Diggins, R. Whiteman, T. D. Clark, R. J. Prance, H. Prance, J. F. Ralph, A. Widom, and Y. N. Srivastava, Physica B 233, 8 (1997).

[24] F. Chiarello, Phys. Lett. A 277, 189 (2000).

[25] S. Zhou, S. Chu, and S. Han, Phys. Rev. B 66, 054527 (2002).

[26] S. Han, Y. Yu, S. Chu, and Z. Wang, Science 293, 1457 (2001)

[27] A. Lupaşcu, C. Vereijis, R. Schouten, C. Harmans, and J. Mooij, e-print cond-mat/0311510.

[28] U. Weiss, Quantum Dissipative Systems (World Scientific, Singapore, 1999).

[29] K. K. Likharev, Dynamics of Josephson Junctions and Circuits (Gordon and Breach, New York, 1986).

[30] R. Migliore and A. Messina, Phys. Rev. B 67, 134505 (2003).

[31] R. Migliore and A. Messina, Int. J. Mod. Phys. B 17, 709 (2003).

[32] E. Almaas and D. Stroud, Phys. Rev. B 65, 134502 (2002).

[33] W. Al-Saidi and D. Stroud, Phys. Rev. B 65, 014512 (2002). 